\title{
Rapid Identification and Verification of Indirubin-Containing Medicinal Plants
}

\author{
Zhigang Hu, ${ }^{1,2}$ Yuan Tu, ${ }^{1,2}$ Ye Xia, ${ }^{1,2}$ Peipei Cheng, ${ }^{1}$ Wei Sun, ${ }^{2}$ Yuhua Shi, ${ }^{2}$ \\ Licheng Guo, ${ }^{1,2}$ Haibo He, ${ }^{2}$ Chao Xiong, ${ }^{1,2}$ Shilin Chen, ${ }^{2}$ and Xiuqiao Zhang ${ }^{1}$ \\ ${ }^{1}$ College of Pharmacy, Hubei University of Chinese Medicine, No. 1 Huangjiahu West Road, Hongshan District, \\ Wuhan 430065, China \\ ${ }^{2}$ Institute of Chinese Materia Medica, China Academy of Chinese Medical Sciences, Beijing 100700, China
}

Correspondence should be addressed to Shilin Chen; slchen@implad.ac.cn and Xiuqiao Zhang; qiaoxzh2000@163.com

Received 19 June 2014; Revised 26 August 2014; Accepted 11 September 2014

Academic Editor: Yi-tao Wang

Copyright (C) 2015 Zhigang Hu et al. This is an open access article distributed under the Creative Commons Attribution License, which permits unrestricted use, distribution, and reproduction in any medium, provided the original work is properly cited.

\begin{abstract}
Indirubin, one of the key components of medicinal plants including Isatis tinctoria, Polygonum tinctorium, and Strobilanthes cusia, possesses great medicinal efficacy in the treatment of chronic myelocytic leukemia (CML). Due to misidentification and similar name, materials containing indirubin and their close relatives frequently fall prey to adulteration. In this study, we selected an internal transcribed spacer 2 (ITS2) for distinguishing these indirubin-containing species from five of their usual adulterants, after assessing identification efficiency of $m a t K, r b c L, p s b A-\operatorname{trnH}$, and ITS2 among these species. The results of genetic distances and neighbor-joining (NJ) phylogenetic tree indicated that ITS2 region is a powerful DNA barcode to accurately identify these indirubin-containing species and discriminate them from their adulterants. Additionally, high performance liquid chromatography (HPLC) was used to verify indirubin in different organs of the above species. The results showed that indirubin had been detected in the leaves of Is. tinctoria, P. tinctorium, S. cusia, and Indigo Naturalis (made from their mixture), but not in their roots, or in the leaves of their adulterants. Therefore, this study provides a novel and rapid method to identify and verify indirubin-containing medicinal plants for effective natural treatment of CML.
\end{abstract}

\section{Introduction}

Chronic myelocytic leukemia (CML) is a malignant cancer that destroys the blood and marrow [1]. In 1967, a group of scientists discovered that the traditional Chinese medicine prescription, Danggui Luhui Wan, which contains 11 Chinese herbal medicines, had a significant curative effect on CML $[2,3]$. More recently, indirubin from the Chinese herbal medicine Indigo Naturalis (Qingdai), prepared from the leaves of Isatis tinctoria, Polygonum tinctorium, and Strobilanthes cusia, was found to be the active ingredient [4-11]. Indirubin has since been found in additional Chinese herbal medicines derived from each of these species, respectively, including Isatidis Folium (the leaf of Is. tinctoria), Polygoni Tinctorii Folium (the leaf of P. tinctorium), and Baphicacanthis Cusiae Rhizoma et Radix (the root and rhizome of S. cusia) [12-14]. However, few studies have determined the presence and containing of indirubin in the above medicinal plants and their adulterants. These adulterants include P. hydropiper, P. chinense, Clerodendrum cyrtophyllum, Indigofera tinctoria, and S. dimorphotricha (Figure 1) and are extremely difficult to discriminate from their true medicinal counterparts morphologically [15-18]. The confusion between true medicinal plants and their adulterants has adverse effects on the clinical efficacy and safety of traditional medicines. It is thus paramount that traditional medicinal herbs and their active components can be reliably and costeffectively discriminated from their false counterparts.

In order to achieve the rapid identification and verification of the indirubin-containing medicinal plants, two important problems must be resolved. First, the original plant species known to contain indirubin must be effectively 


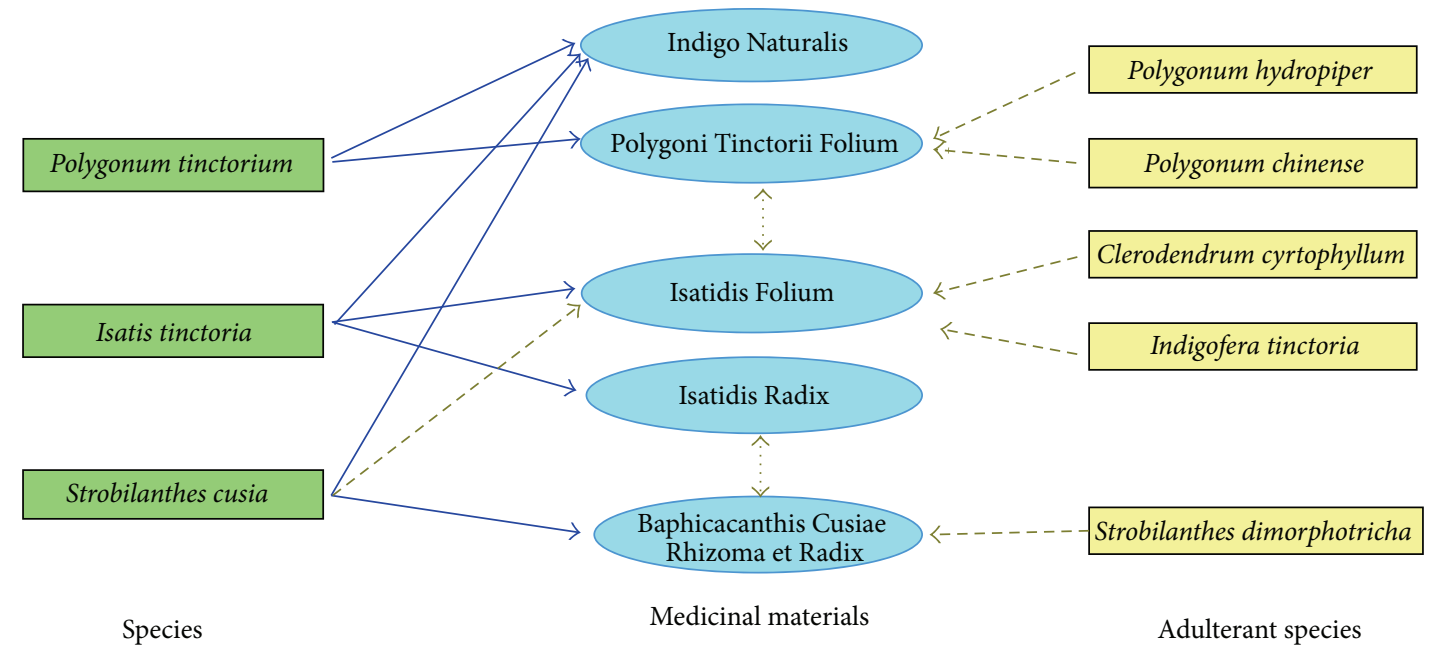

$\longrightarrow$ Means source of medicinal materials

$-\rightarrow$ Means relationship of adulterants

FIGURE 1: The relationship among indirubin-containing medicinal materials (plant organs/formulations), their plants of origin, and adulterants.

discriminated from their adulterants. DNA barcoding technology offers the best technique for this to date, involving specific amplification of a short, standardized DNA fragment with universal primers across multiple samples $[19,20]$. In recent years, several candidate DNA regions, such as matK, $r b c L, p s b A-t r n H$, and ITS/ITS2, have been assessed for their potential as DNA barcodes in plants [21-25]. Analysis of 50,790 plant ITS2 sequences revealed this region to be highly effective in discriminating medicinal plants and their closely related species $[23,26]$, more so than $m a t K, r b c L$, and $p s b A$ $\operatorname{trn} H$. As such, ITS sequences have been recommended as the core barcode region for seed plants [27]. Here, we reveal the first rapid and effective method to reliably discriminate indirubin-containing species in traditional medicinal formulations from their adulterants, using ITS2 barcoding. Secondly, it is necessary that the different plant organs comprising traditional medicinal materials (Figure 1) can be analysed for indirubin levels effectively. High performance liquid chromatography (HPLC) offers an effective screening technique in this regard.

\section{Materials and Methods}

2.1. Plant Materials. A total of 57 samples from 8 species were gathered from various geographical areas in China, as detailed in Table 1 . All the specimens were carefully visually identified using standard expert identification parameters at the Institute of Medicinal Plant Development (IMPLAD), Chinese Academy of Medical Sciences. The specimens were deposited in the herbarium of the Hubei University of Chinese Medicine.
2.2. DNA Extraction, PCR Amplification, and Sequencing. Samples comprising 30 40 mg of dried leaves or $60 \sim 70 \mathrm{mg}$ of roots were crushed into powder in $2 \mathrm{~mL}$ microfuge tubes at $30 \mathrm{~Hz}$ using stainless steel ball milling for $1 \mathrm{~min}$. Total genomic DNA was extracted using a Plant Genomic DNA Kit (Tiangen Biotech Co., China) with modifications as follows. Initial incubation was at $65^{\circ} \mathrm{C}$ in $750 \mu \mathrm{L}$ GP1 (Tiangen) buffer for $1 \mathrm{~h}$ for dried leaves or $5 \mathrm{~h}$ for roots and rhizomes. The remaining steps followed the manufacturer's protocol.

For matK, rbcL, psbA-trnH, and ITS2 DNA barcodes, universal primers and general PCR reaction conditions were used as presented in Table S1 (see Table S1 in Supplementary Material available online at http://dx.doi.org/10.1155/ $2015 / 484670)[23,28]$. PCRs were in a $25 \mu \mathrm{L}$ reaction mixture, containing 30-100 ng of genomic DNA template, $12.5 \mu \mathrm{L} 2 \times$ Tag PCR Master Mix (Aidlab Biotechnologies Co., China), and $1 \mu \mathrm{L}$ of forward and reverse primers $(2.5 \mu \mathrm{mol} / \mathrm{L})$. After PCR, a $4 \mu \mathrm{L}$ aliquot was examined by $0.5 \%$ TBE agarose gel electrophoresis, and purified PCR products were sequenced in both directions using the primers used for PCR on an ABI3730XL sequencer (Applied Biosystems Co., USA).

2.3. Cloning and Sequencing of the ITS2 Region. The ITS2 PCR products of S. cusia and S. dimorphotricha were unsuccessfully directly sequenced. Therefore, purified products (TIANquick Midi Purification Kit; Tiangen Biotech Co., China) were ligated into the pMD18-T vector (Takara Biotech Co., China) and transformed into E. coli DH5 cells using standard recombinant DNA techniques. Positive transformants were selected on LB containing $0.1 \mathrm{mg} / \mathrm{mL}$ ampicillin and confirmed with colony PCR using the above PCR conditions. 
TABLE 1: Detailed description of all the samples in this study.

\begin{tabular}{|c|c|c|c|c|}
\hline Species & Medicinal part & Locality & Voucher number & GenBank number of ITS2 \\
\hline Isatis tinctoria & Leaves & Hebei, China & YC0021MT02 & KJ939152 \\
\hline Is. tinctoria & Leaves & Anhui, China & YC0021MT09 & KJ939157 \\
\hline Is. tinctoria & Leaves & Chongqing, China & YC0021MT10 & KJ939158 \\
\hline Is. tinctoria & Leaves & Chongqing, China & YC0021MT12 & KJ939159 \\
\hline Is. tinctoria & Leaves & Chongqing, China & YC0021MT13 & KJ939160 \\
\hline Is. tinctoria & Leaves & Yunnan, China & YC0021MT14 & KJ939161 \\
\hline Is. tinctoria & Leaves & Beijing, China & YC0021MT15 & KJ939162 \\
\hline Is. tinctoria & Leaves & Beijing, China & YC0021MT04 & KJ939154 \\
\hline Is. tinctoria & Leaves & Beijing, China & YC0021MT05 & KJ939155 \\
\hline Is. tinctoria & Leaves & Beijing, China & YC0021MT06 & KJ939156 \\
\hline Is. tinctoria & Roots & Sichuan, China & YC0021MT01 & KJ939151 \\
\hline Is. tinctoria & Roots & Hebei, China & YC0021MT03 & KJ939153 \\
\hline Is. tinctoria & Roots & Beijing, China & YC0021MT20 & KJ939163 \\
\hline Is. tinctoria & Roots & Beijing, China & YC0021MT21 & KJ939164 \\
\hline Is. tinctoria & Roots & Beijing, China & YC0021MT22 & KJ939165 \\
\hline Is. tinctoria & Roots & Beijing, China & YC0021MT23 & KJ939166 \\
\hline Is. tinctoria & Roots & Sichuan, China & YC0021MT29 & KJ939167 \\
\hline Is. tinctoria & Roots & Hubei, China & YC0021MT30 & KJ939168 \\
\hline Polygonum tinctorium & Leaves & Beijing, China & YC0390MT04 & KJ939177 \\
\hline P. tinctorium & Leaves & Beijing, China & YC0390MT05 & KJ939178 \\
\hline P. tinctorium & Leaves & Beijing, China & YC0390MT01 & KJ939174 \\
\hline P. tinctorium & Leaves & Beijing, China & YC0390MT07 & KJ939179 \\
\hline P. tinctorium & Leaves & Beijing, China & YC0390MT09 & KJ939181 \\
\hline P. tinctorium & Leaves & Fujian, China & PS2901MT01 & FJ503014 \\
\hline Strobilanthes cusia & Leaves & Guangdong, China & YC0389MT01 & KJ939116-KJ939119 \\
\hline S. cusia & Leaves & Chongqing, China & YC0389MT02 & KJ939109-KJ939112 \\
\hline S. cusia & Leaves & Chongqing, China & YC0389MT03 & KJ939113-KJ939115 \\
\hline S. cusia & Leaves & Hainan, China & YC0389MT04 & KJ939125-KJ939127, KJ939104 \\
\hline S. cusia & Leaves & Yunnan, China & YC0389MT07 & KJ939139, KJ939140 \\
\hline S. cusia & Leaves & Yunnan, China & YC0389MT08 & KJ939141-KJ939143 \\
\hline S. cusia & Leaves & Guangxi, China & YC0389MT10 & KJ939105, KJ939137, KJ939138 \\
\hline S. cusia & Leaves & Fujian, China & YC0389MT11 & KJ939133-KJ939136 \\
\hline S. cusia & Leaves & Guangxi, China & YC0389MT12 & KJ939120-KJ939122 \\
\hline S. cusia & Leaves & Guangxi, China & YC0389MT13 & KJ939123, KJ939124 \\
\hline S. cusia & Roots and rhizomes & Hainan, China & YC0389MT05 & KJ939128-KJ939130 \\
\hline S. cusia & Roots and rhizomes & Hainan, China & YC0389MT06 & KJ939131, KJ939132 \\
\hline S. cusia & Roots and rhizomes & Guangdong, China & YC0389MT14 & KJ939106-KJ939108 \\
\hline Polygonum hydropiper & Leaves & Guangdong, China & YC0509MT01 & KJ939169 \\
\hline P. hydropiper & Leaves & Guangdong, China & YC0509MT02 & KJ939170 \\
\hline P. hydropiper & Leaves & Guangdong, China & YC0509MT03 & KJ939171 \\
\hline P. hydropiper & Leaves & Guangdong, China & YC0509MT04 & KJ939172 \\
\hline P. hydropiper & Leaves & Guangdong, China & YC0509MT05 & KJ939173 \\
\hline Polygonum chinense & Leaves & Guangxi, China & YC0510MT01 & KJ939182 \\
\hline P. chinense & Leaves & Guangxi, China & YC0510MT02 & KJ939183 \\
\hline P. chinense & Leaves & Guangdong, China & YC0510MT03 & KJ939184 \\
\hline P. chinense & Leaves & Guangdong, China & YC0510MT04 & KJ939185 \\
\hline Clerodendrum cyrtophyllum & Leaves & Guangxi, China & YC0508MT01 & KJ939144 \\
\hline
\end{tabular}


TABLe 1: Continued.

\begin{tabular}{|c|c|c|c|c|}
\hline Species & Medicinal part & Locality & Voucher number & GenBank number of ITS2 \\
\hline C. cyrtophyllum & Leaves & Guangxi, China & YC0508MT02 & KJ939145 \\
\hline C. cyrtophyllum & Leaves & Guangxi, China & YC0508MT03 & KJ939146 \\
\hline C. cyrtophyllum & Leaves & Guangxi, China & YC0508MT04 & KJ939147 \\
\hline Strobilanthes dimorphotricha & Leaves & Guangdong, China & YC0511MT01 & KJ939187, KJ939188, KJ939191 \\
\hline S. dimorphotricha & Leaves & Guangdong, China & YC0511MT02 & KJ939189, KJ939190 \\
\hline S. dimorphotricha & Leaves & Guizhou, China & YC0511MT03 & KJ939192, KJ939193 \\
\hline Indigofera tinctoria & Leaves & Guangxi, China & YC0707MT01 & KJ939148 \\
\hline In. tinctoria & Leaves & Guangxi, China & YC0707MT02 & KJ939149 \\
\hline In. tinctoria & Leaves & Guangxi, China & YC0707MT03 & KJ939150 \\
\hline In. tinctoria & Leaves & Guangxi, China & PS0251MT02 & GU217625 \\
\hline
\end{tabular}

Four positive clones from each sample were sequenced on an ABI3730XL sequencer. In total, 40 clones from 13 samples of $S$. cusia and 7 clones from 3 samples of $S$. dimorphotricha (excluding fungal sequences) were obtained.

2.4. Sequence Analyses. Sequence editing and contig assembly were performed using CodonCode Aligner v4.25 (CodonCode Co., USA). The ITS2 region was obtained based on the HMMER annotation method to remove the $5.8 \mathrm{~S}$ and $28 \mathrm{~S}$ sections at both ends of the sequences [29]. Obtained DNA sequences were aligned and the intraspecific variation and interspecific divergence calculated by Kimura two-parameter method. Phylogenetic trees were constructed using the Neighbor-Joining method with molecular evolutionary genetics analysis (MEGA) software version 5.0 [30].

2.5. HPLC Analyses. The reference standard of indirubin was purchased from Shanghai Yuanye Bio-Technology Company (HPLC-tested purity $>98 \%$ ). $1.25 \mathrm{mg}$ of indirubin was dissolved in $\mathrm{N}, \mathrm{N}$-dimethyl formamide in a $25 \mathrm{~mL}$ volumetric flask. Five milliliters of solution was transferred to another $50 \mathrm{~mL}$ volumetric flask containing N,N-dimethyl formamide to make a standard stock solution of $5 \mu \mathrm{g} / \mathrm{mL}$ indirubin. Two-three replicate samples from each tested plant organ and species were randomly sampled for indirubin content. Specifically, $75 \mathrm{mg}$ of powdered crude materials was sonicated in $10 \mathrm{~mL}$ of $\mathrm{N}, \mathrm{N}$-dimethyl formamide for $30 \mathrm{~min}$ at room temperature and filtered. A $20 \mu \mathrm{L}$ aliquot of the filtrate was applied to a HPLC column (Angilent TC-C ${ }_{18}, 5.0 \mu \mathrm{m}, 4.6 \mathrm{~mm}$ $\times 250 \mathrm{~mm}$ ). The optimum separation of HPLC was carried out with a mobile phase composed of methanol-water $(75: 25$, $\mathrm{v} / \mathrm{v}$ ) at a flow-rate of $1 \mathrm{~mL} / \mathrm{min}$. Peaks were detected at a wavelength and column temperature of $290 \mathrm{~nm}$ and $25^{\circ} \mathrm{C}$, respectively.

\section{Results}

3.1. Efficiency of Amplification and Identification for Four Candidate Barcodes. For all eight species tested, 24 samples were selected randomly for amplification efficiency. The efficiency of amplification of matK, rbcL, psbA-trnH, and ITS2 was $62.5 \%, 79.2 \%, 100 \%$, and $100 \%$, respectively. Highquality bidirectional sequences were obtained for all PCR products. All the GenBank No. were listed in Table S2 and Table 1. Alignment revealed no interspecific divergence between S. cusia and S. dimorphotricha using both $p s b A$-trnH and $r b c L$. In addition, mat $K$ had low amplification efficiency $(62.5 \%)$, making this less applicable for barcoding of these species. In comparison with the other barcodes, all 24 samples were successfully classified into eight species using the ITS2 sequence. Therefore, only the ITS2 barcode was used for further analysis.

3.2. Measurement of DNA Divergence for ITS2. Song et al. (2012) used sequence-tagged pyrosequencing and genomewide analyses to describe intragenomic variations of ITS2 regions from 178 plant species. This study defined "major variants" as any variant whose relative variant abundance (RVA) was greater than 5\% [31]. In this study we obtained 40 clones of S. cusia and only 2 of them (KJ939104, KJ939105) showed significant differences when compared with the other sequences. The remaining 38 sequences were considered major variants of ITS2 in this paper.

In this study, 86 sequences of ITS2 were obtained from all samples. Two ITS2 sequences (EU196919, JN235085) of $P$. tinctorium were downloaded from GenBank. The sequence length, GC average content, haplotype number and number of variable sites in each species (MEGA 5.0 software) are presented in Table 2. ITS2 sequence length ranged from $191 \mathrm{bp}$ to $263 \mathrm{bp}$ and GC average content ranged from $45.9 \%$ to $73.6 \%$. The GC average contents of clones of S. cusia (73.6\%) and $S$. dimorphotricha $(73.0 \%)$ were at least $4 \%$ greater than those of the other species. Based on the variable sites, Is. tinctoria, $P$. tinctorium, and S. cusia were divided into 5, 1 , and 15 haplotypes, respectively.

Interspecific and intraspecific distances using Kimura two-parameter method are shown in Table 3. The maximum intraspecific distances of Is. tinctoria, P. tinctorium, and $S$. cusia were $0.027,0.000$, and 0.036 , respectively, while the minimum interspecific distance was 0.401 . Furthermore, the minimum interspecific distances between any one of these 
TABLE 2: Sequence characteristics of the related species.

\begin{tabular}{|c|c|c|c|c|}
\hline Species/(number of sequences) & Length of ITS2 (bp) & GC average content (\%) & Number of haplotypes & Number of variable sites \\
\hline Is. tinctoria (18) & 191 & 56.7 & 5 & 5 \\
\hline P. tinctorium (8) & 245 & 68.2 & 1 & 1 \\
\hline S. cusia (38) & $230 \sim 235$ & 73.6 & 15 & 20 \\
\hline P. hydropiper (5) & 244 & 68.9 & 1 & 1 \\
\hline P. chinense (4) & 263 & 65.8 & 1 & 1 \\
\hline C. cyrtophyllum (4) & 224 & 56.7 & 1 & 1 \\
\hline S. dimorphotricha (7) & $224 \sim 233$ & 73.0 & 6 & 13 \\
\hline In. tinctoria (4) & 219 & 45.9 & 1 & 1 \\
\hline
\end{tabular}

TABLE 3: Data of interspecific and intraspecific distances of the related species.

\begin{tabular}{lc}
\hline Parameter & Range \\
\hline Intraspecific distances of Is. tinctoria & $0.000 \sim 0.027$ \\
Intraspecific distances of $P$. tinctorium & 0.000 \\
Intraspecific distances of S. cusia & $0.000 \sim 0.036$ \\
Interspecific distance among the above three species & $0.401 \sim 0.684$ \\
Interspecific distance between Is. tinctoria and its adulterants & $0.514 \sim 0.684$ \\
Interspecific distance between $P$. tinctorium and its adulterants & $0.025 \sim 0.755$ \\
Interspecific distance between S. cusia and its adulterants & $0.065 \sim 0.931$ \\
\hline
\end{tabular}

three species and its adulterants were $0.514,0.025$, and 0.065 , respectively.

3.3. Identification of ITS2 Using NJ Tree. Phylogenetic analysis demonstrated that every species clustered into their own clade, supported with at least $81 \%$ bootstrapping (Figure 2 ). In addition, three closely related species of the genus Polygonum were strongly supported $(99 \%, 81 \%$ and $100 \%$ bootstrap, resp.), and clustered into a larger branch with $97 \%$ bootstrap. All cloned sequences of S. cusia (96\% bootstrap) and S. dimorphotricha (92\% bootstrap) in Acanthaceae family formed a larger group (93\% bootstrap).

3.4. Detection of Indirubin in Crude Drugs and Their Adulterants. HPLC detected indirubin in the leaves of Is. tinctoria, P. tinctorium, S. cusia, and Indigo Naturalis (Figure 3). Indirubin was not detected in the roots and rhizomes of these three taxa or in the leaves of their adulterants $(P$. hydropiper, P. chinense, C. cyrtophyllum, In. tinctoria, and S. dimorphotricha).

\section{Discussion}

In previous studies, the identification methods of medicinal plants including Is. tinctoria, P. tinctorium, and S. cusia have primarily focused on characterization of morphology, chromatographic fingerprints, and microstructures [32-34]. However, these methods all have their disadvantages. The recent, rapid development of DNA molecular marker techniques provides a powerful tool for the accurate identification of medicinal materials. In recent years, DNA barcoding has been successfully employed in species identification of medicinal herbs, with the ITS2 barcode exhibiting remarkable stability and accuracy in this field. ITS/ITS2 regions were demonstrated to successfully distinguish Corni Fructus (the flesh of Cornus officinalis) from its adulterants [35]. Xin et al. (2013) presented the ITS2 barcode as a powerful tool for tracing Goji (the fruit of Lycium barbarum) [36], while it was also used to accurately identify Ephedrae Herba (the stem taken from three species of Ephedra) and their closely related species [37]. Consequently, the rapidly developing DNA barcoding can effectively supplement the traditional identification methods. In this study, the ITS2 region was selected from four candidate barcodes to identify three species and their adulterants because of $100 \%$ amplification efficiency herein, high interspecific divergence, and low intraspecific variation. Based on the ITS2 barcode, the maximum intraspecific distance of the three species (Is. tinctoria, $P$. tinctorium and S. cusia) was less than the minimum interspecific distance, not only among the three species, but also among each species and its adulterants. Furthermore, the NJ tree indicated that Is. tinctoria, P. tinctorium, and $S$. cusia were clustered into their own monophyletic group, separated from the other species. Moreover, NJ tree analysis using ITS2 reliably distinguished individuals of the genus Polygonum and the family of Acanthaceae, supporting the powerful identification ability of ITS2 barcode in plants. 


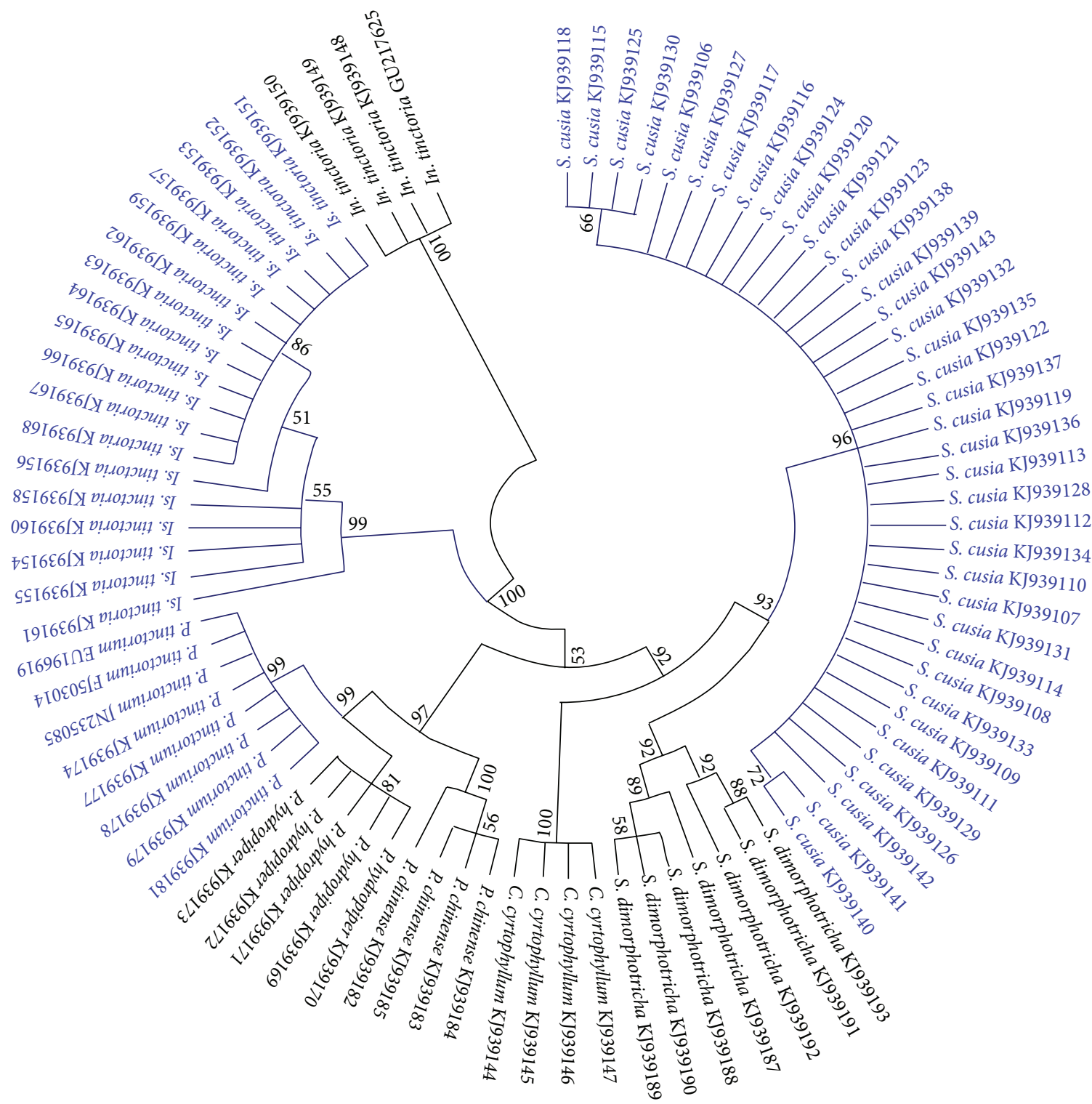

FIGURE 2: Phylogenetic tree of all the medicinal plants containing indirubin and their adulterants constructed with the ITS2 sequences using NJ method (Bootstrap scores $\geq 50 \%$ ). The samples marked with blue represent the medicinal plants containing indirubin, and the others represent their adulterants.

Therefore, both the results of nearest distance method and NJ tree strongly support that ITS2 as DNA barcode can successfully distinguish Is. tinctoria, P. tinctorium, and S. cusia from each other and from their respective adulterants.

The demonstrated anticancer function of indirubin in the treatment of Chronic myelocytic leukemia (CML), warrants its further investigation and ability to be identified accurately in natural medicines. In this study, HPLC detection found that indirubin could only be detected in the leaves of Is. tinctoria, P. tinctorium, S. cusia and Indigo Naturalis. Meanwhile it could not be found in the root tissues of these species, or in the adulterant species tested herein. These results confirm that Is. tinctoria, P. tinctorium, and S. cusia cannot be replaced by their adulterants as indirubin-containing tinctures. And not only that, the species used for traditional medicinal herbs can be extremely disordered because of a general divergence in regional customs and species identification abilities [38]. All of these strongly supported the need for accurate discrimination of these ineffective false "pseudo"medicines.

\section{Conclusion}

Together with HPLC detection of indirubin in various organs, ITS2 DNA barcoding enables the rapid, efficient, and costeffective discrimination of the truly effective preparations of medicinal plants from their noneffective organs and adulterants that do not contain indirubin. This provides an efficient 


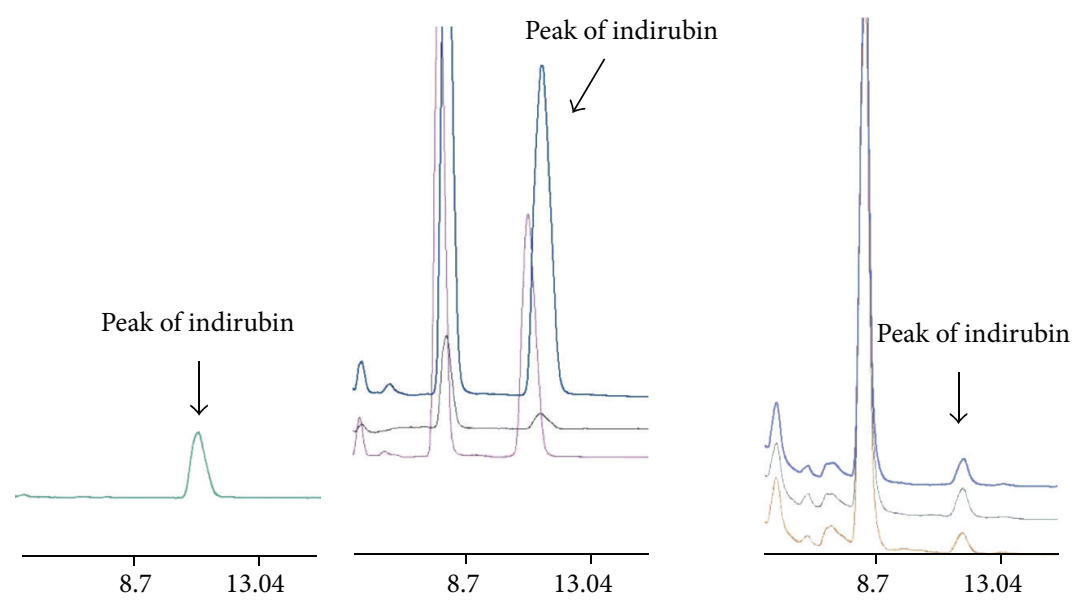

(a) Reference substance

(b) Indigo Naturalis

(c) Leaves of P. tinctorium

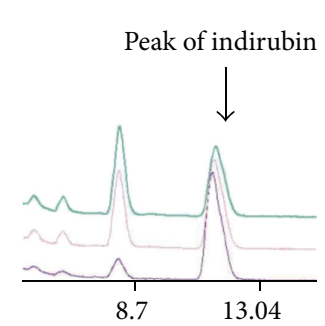

(d) Leaves of Is. tinctoria

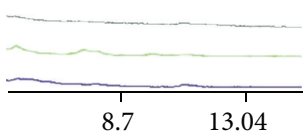

(e) Roots of Is. tinctoria

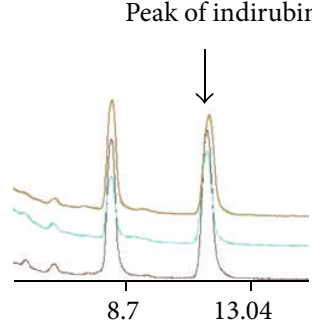

(f) Leaves of S. cusia

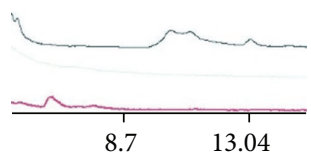

(g) Roots and Rhizomes of S. cusia

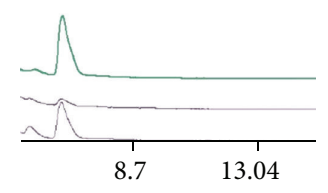

(h) Leaves of C. cyrtophyllum

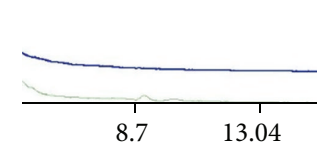

(i) Leaves of P. chinense

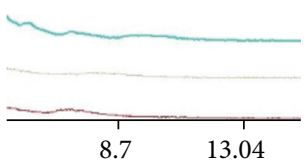

(j) Leaves of In. tinctoria

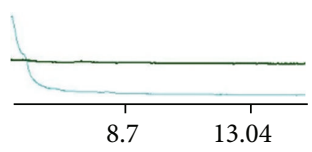

(k) Leaves of $S$. dimorphotricha

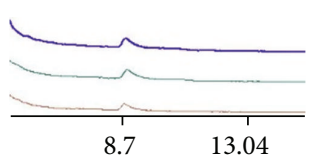

(1) Leaves of P. hydropiper

FiguRE 3: HPLC profiles of N,N-dimethyl formamide extract prepared from different organs of medicinal plants and five of their usual adulterants. Each profile was made up of two-three replicate samples from each tested plant organ and species.

and new method to verify indirubin-containing medicines for the natural treatment of CML.

\section{Conflict of Interests}

All the authors declare that there is no conflict of interests regarding the publication of this paper.

\section{Authors' Contribution}

Zhigang $\mathrm{Hu}$ and Yuan $\mathrm{Tu}$ were equal contributors to this paper.

\section{Acknowledgments}

This study was supported by the key project of the National Natural Science Foundation of China (no. 81130069) and the National Science and Technology Major Project for
"Major New Drugs Innovation and Development" (no. 2014ZX09304307001).

\section{References}

[1] H. M. Kantarjian, A. Deisseroth, R. Kurzrock, Z. Estrov, and M. Talpaz, "Chronic myelogenous leukemia: a concise update," Blood, vol. 82, no. 3, pp. 691-703, 1993.

[2] The Sixth Groups of Chinese Academy of Medical Sciences, "The study on anti-leukemia of Dang Gui Lu Hui Wan," Bulletin of Medical Research, no. 7, pp. 28-30, 1975.

[3] R. Hoessel, S. Leclerc, J. A. Endicott et al., "Indirubin, the active constituent of a Chinese antileukaemia medicine, inhibits cyclin-dependent kinases," Nature Cell Biology, vol. 1, no. 1, pp. 60-67, 1999.

[4] S. Nam, R. Buettner, J. Turkson et al., "Indirubin derivatives inhibit Stat3 signaling and induce apoptosis in human cancer cells," Proceedings of the National Academy of Sciences of the United States of America, vol. 102, no. 17, pp. 5998-6003, 2005. 
[5] M. Z. Ma, "The observation on curative effect of treating chronic myelogenous leukemia of Indigo Naturalis," Chinese Journal of Medicine, vol. 4, pp. 22-25, 1979.

[6] G. Y. Wu, F. D. Fang, J. Z. Liu et al., "Research on the principle using indigo for the curative effect of chronic myelogenous leukemia (1): the influence on the DNA, RNA and protein synthesis of peripheral leukemia cells to the person," National Medical Journal of China, vol. 60, no. 8, pp. 451-454, 1980.

[7] G. Y. Wu, J. Z. Liu, D. F. Fang, and J. Zuo, "Research on the principle using indigo for the curative effect of chronic myelogenous leukemia: identification on the function and bond types of the combination of indigo and DNA," Science in China $B$, no. 5, pp. 436-443, 1982.

[8] Z. Xiao, Y. Hao, B. Liu, and L. Qian, "Indirubin and Meisoindigo in the treatment of chronic myelogenous leukemia in China," Leukemia \& Lymphoma, vol. 43, no. 9, pp. 1763-1768, 2002.

[9] D. Marko, S. Schätzle, A. Friedel et al., "Inhibition of cyclindependent kinase 1 (CDK1) by indirubin derivatives in human tumour cells," British Journal of Cancer, vol. 84, no. 2, pp. 283289, 2001.

[10] Q. W. Wu, Z. L. Ge, Y. Gao, and L. Zhang, "Inhibitory effect of indirubin on growth of some cancer cells and its mechanism," Tianjin Journal of Traditional Chinese Medicine, vol. 25, no. 1, pp. 55-58, 2008.

[11] J. Liu, C. Zeng, L. P. Hu, and H. C. Chen, "Mechanism of proliferative inhibition of indirubin on K562 cells," Guiding Journal of Traditional Chinese Medicine and Pharmacy, vol. 15, no. 2, pp. 10-12, 2009.

[12] H.-C. Hou and S.-Z. Liang, "Determination of indirubin and indigo in Baphicacanthus cusia (Nees) Bremek by HPLC," Journal of Chinese Medicinal Materials, vol. 29, no. 7, pp. 681682, 2006.

[13] Y. X. Zhan, Q. Fu, R. Duan et al., "Study on determination of indigo and indirubin contents and HPLC fingerprints of Isatidis Folium from various sources," Modern Chinese Medicine, vol.13, no. 6, pp. 20-23, 2011.

[14] Chinese Pharmacopoeia Commission, Pharmacopoeia of the People's Republic of China, vol. 1, Chinese Medical Science and Technology Press, Beijing, China, 2010.

[15] Z. Y. Sun, H. M. Luo, and S. L. Chen, "Molecular identification of Isatidis Folium and its adulterants by ITS2 sequences," World Science and Technology /Modernization of Traditional Chinese Medicine and Materia Medica, vol. 13, no. 2, pp. 395-399, 2011.

[16] B.-Z. He, J.-J. Qin, Y.-L. Zhu, and Y.-K. Liao, "Identification characters of leaf morphological and venation pattern of Baphicacanthus cusia with its confused herb Clerodendrum cyrtophyllum," Journal of Chinese medicinal materials, vol. 35, no. 3, pp. 385-391, 2012.

[17] G. Q. Li, Z. T. Wang, X. B. Li, and G. J. Xu, "Determination of nrDNA ITS sequence of Liaodaqingye (Folium Polygoni Tinctorii) and its counterfeit," Chinese Wild Plant Resources, vol. 20, no. 3, pp. 43-46, 2001.

[18] H. Xu, R. Z. Du, P. M. Yang, Y. Zeng, P. J. Chu, and Z. L. Shi, "Identification of Baphicacanthis Cusiae Rhizoma et Radix and its adulterants," Research and Practice of Chinese Medicines, vol. 17, no. 6, pp. 54-55, 2003.

[19] P. D. N. Hebert, A. Cywinska, S. L. Ball, and J. R. Waard, "Biological identifications through DNA barcodes," Proceedings of the Royal Society B: Biological Sciences, vol. 270, no. 1512, pp. 313-321, 2003.

[20] R. Lahaye, M. Van Der Bank, D. Bogarin et al., "DNA barcoding the floras of biodiversity hotspots," Proceedings of the National
Academy of Sciences of the United States of America, vol. 105, no. 8, pp. 2923-2928, 2008.

[21] M. W. Chase, N. Salamin, M. Wilkinson et al., "Land plants and DNA barcodes: short-term and long-term goals," Philosophical Transactions of the Royal Society B: Biological Sciences, vol. 360, no. 1462, pp. 1889-1895, 2005.

[22] CBOL Plant Working Group, "A DNA barcode for land plants," Proceedings of the National Academy of Sciences of the United States of America, vol. 106, no. 31, pp. 12794-12797, 2009.

[23] S. Chen, H. Yao, J. Han et al., "Validation of the ITS2 region as a novel DNA barcode for identifying medicinal plant species," PLoS ONE, vol. 5, no. 1, Article ID e8613, 2010.

[24] D. Hou, J. Song, L. Shi et al., "Stability and accuracy assessment of identification of traditional chinese materia medica using DNA barcoding: a case study on Flos Lonicerae Japonicae," BioMed Research International, vol. 2013, Article ID 549037, 8 pages, 2013.

[25] X. W. Li, Y. Yang, R. J. Henry, M. Rossetto, Y. T. Wang, and S. L. Chen, "Plant DNA barcoding: from gene to genome," Biological Reviews, 2014.

[26] H. Yao, J. Song, C. Liu et al., "Use of ITS2 region as the universal DNA barcode for plants and animals," PLOS ONE, vol. 5, no. 10, Article ID e13102, 2010.

[27] D.-Z. Li, L.-M. Gao, H.-T. Li et al., "Comparative analysis of a large dataset indicates that internal transcribed spacer (ITS) should be incorporated into the core barcode for seed plants," Proceedings of the National Academy of Sciences of the United States of America, vol. 108, no. 49, pp. 19641-19646, 2011.

[28] S. L. Chen, Molecular Identification of Chinese Materia Medica Using DNA Barcodes, People's Publishing House, Beijing, China, 2010.

[29] A. Keller, T. Schleicher, J. Schultz, T. Müller, T. Dandekar, and M. Wolf, "5.8S-28S rRNA interaction and HMM-based ITS2 annotation,” Gene, vol. 430, no. 1-2, pp. 50-57, 2009.

[30] K. Tamura, D. Peterson, N. Peterson, G. Stecher, M. Nei, and S. Kumar, "MEGA5: molecular evolutionary genetics analysis using maximum likelihood, evolutionary distance, and maximum parsimony methods," Molecular Biology and Evolution, vol. 28, no. 10, pp. 2731-2739, 2011.

[31] J. Song, L. Shi, D. Li et al., "Extensive pyrosequencing reveals frequent intra-genomic variations of internal transcribed spacer regions of nuclear ribosomal DNA," PLoS ONE, vol. 7, no. 8, Article ID e43971, 2012.

[32] P. Zou, Y. Hong, and H. L. Koh, "Chemical fingerprinting of Isatis indigotica root by RP-HPLC and hierarchical clustering analysis," Journal of Pharmaceutical and Biomedical Analysis, vol. 38, no. 3, pp. 514-520, 2005.

[33] Y. Shi, Z. Xie, R. Wang, S. Huang, Y. Li, and Z. Wang, "Chromatographic fingerprint study on water-soluble extracts of radix isatidis, folium isatidis, and their preparations by HPLC-DAD technique," Journal of Liquid Chromatography and Related Technologies, vol. 36, no. 1, pp. 80-93, 2012.

[34] S. Nie, Y. Zu, L. Zhang, and Y. Pei, "A comparative study on microstructure between Isatis indigotica Fort. and Baphicacanthus cusia (Nees) Bremek," Agricultural Science \& TechnologyHunan, vol. 13, no. 4, pp. 763-826, 2012.

[35] D.-Y. Hou, J.-Y. Song, H. Yao et al., "Molecular identification of Corni fructus and its adulterants by ITS/ITS2 sequences," Chinese Journal of Natural Medicines, vol. 11, no. 2, pp. 121-127, 2013. 
[36] T. Y. Xin, H. Yao, H. H. Gao et al., "Super food Lycium barbarum (Solanaceae) traceability via an internal transcribed spacer 2 barcode," Food Research International, vol. 54, no. 2, pp. 16991704, 2013.

[37] X. H. Pang, J. Y. Song, H. B. Xu, and H. Yao, "Using ITS2 barcode to identify Ephedrae herba," China Journal of Chinese Materia Medica, vol. 37, no. 8, pp. 1118-1121, 2012.

[38] Z. Zhao, J. P. S. Yuen, J. Wu, T. Yu, and W. Huang, "A systematic study on confused species of Chinese materia medica in the Hong Kong market," Annals of the Academy of Medicine Singapore, vol. 35, no. 11, pp. 764-769, 2006. 


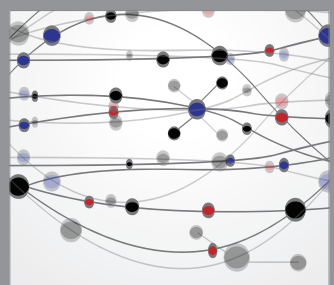

The Scientific World Journal
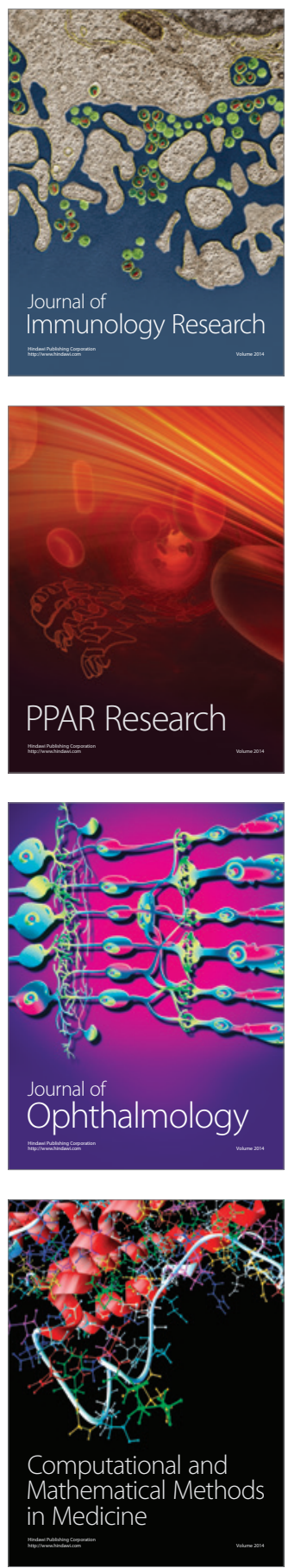

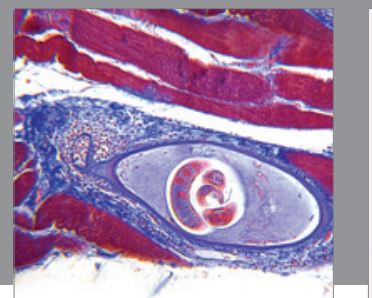

Gastroenterology

Research and Practice
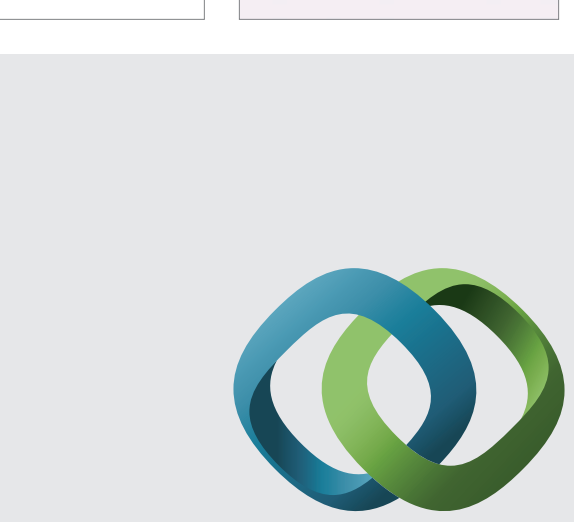

\section{Hindawi}

Submit your manuscripts at

http://www.hindawi.com
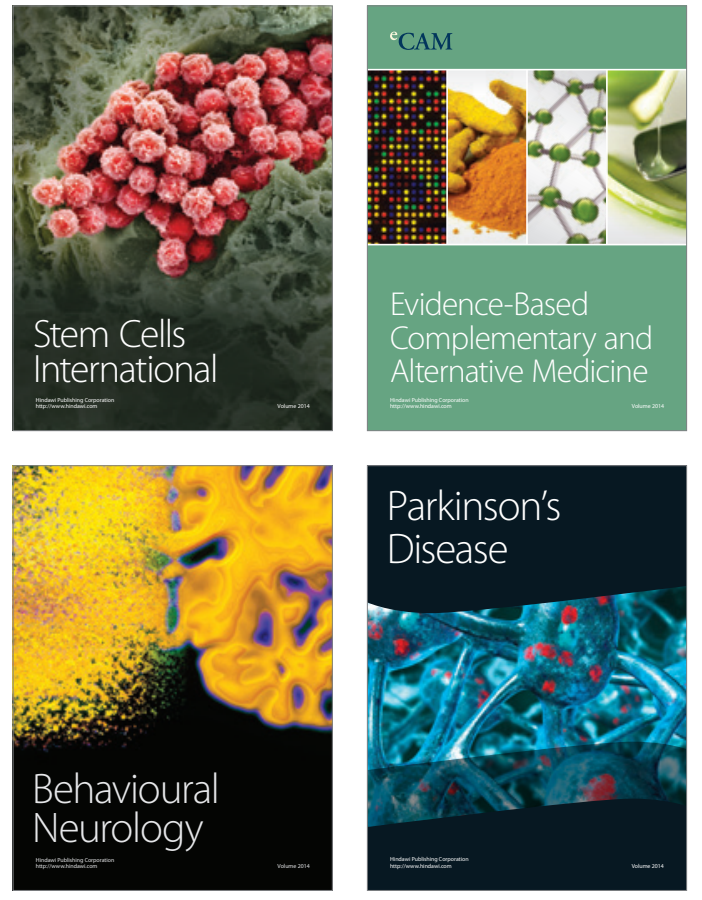
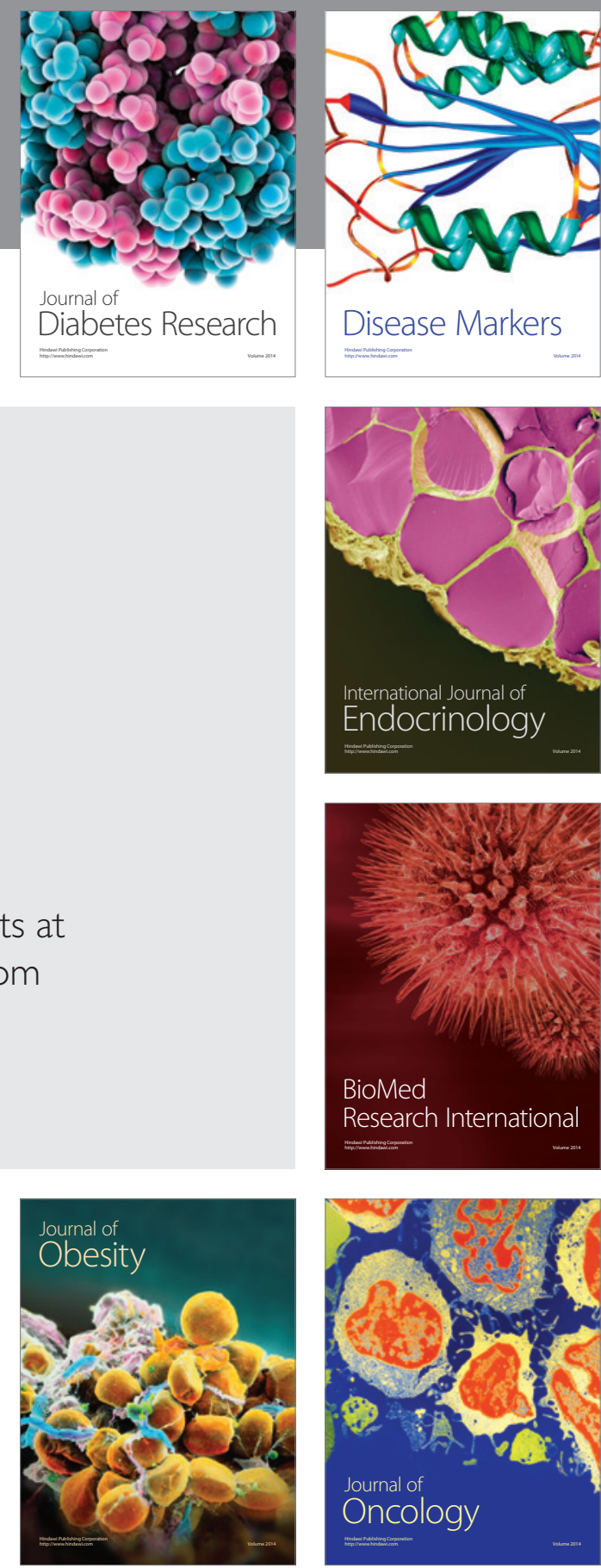

Disease Markers
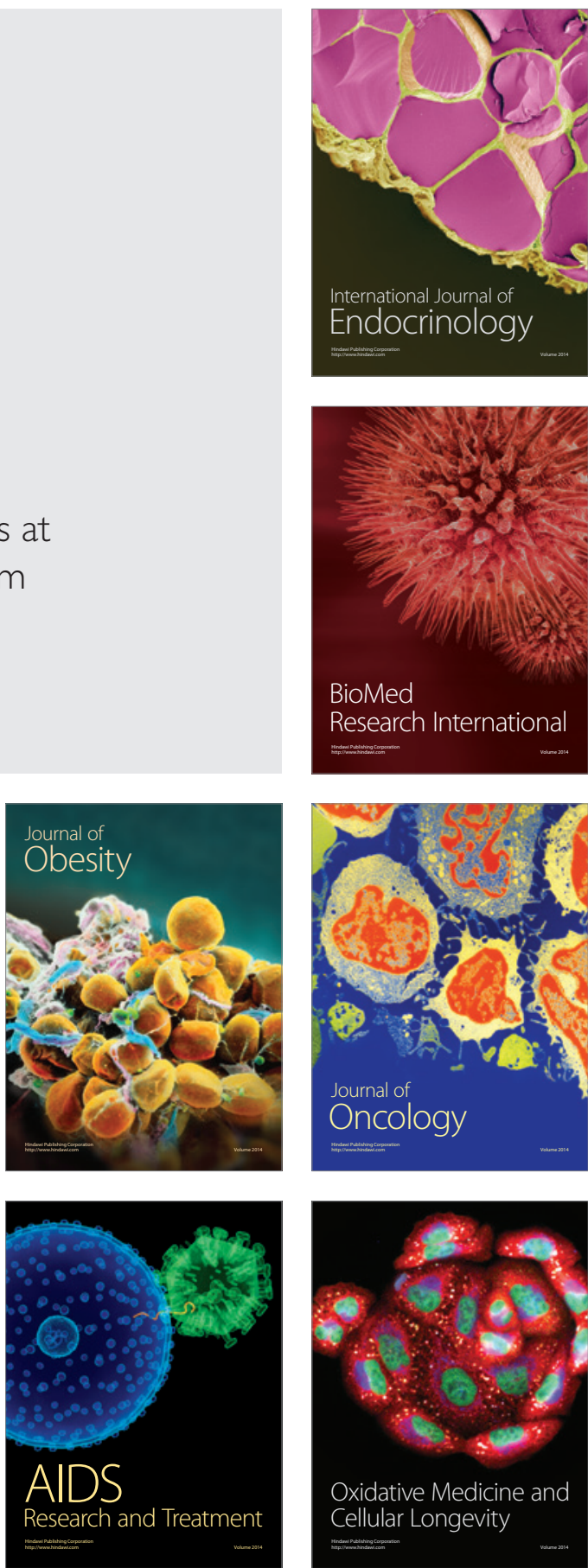